\title{
Bayes Estimate of Primary Threshold in Cluster-wise fMRI Inferences
}

\author{
Yunjiang Ge ${ }^{1}$, Stephanie Hare ${ }^{2}$, Gang Chen ${ }^{3}$, James Waltz ${ }^{2}$, Peter \\ Kochunov $^{2}$, L. Elliot Hong ${ }^{2}$, and Shuo Chen ${ }^{2,4}$ \\ ${ }^{1}$ Department of Mathematics, University of Maryland-College Park, 4176 Campus Dr, College \\ Park, MD 20742, USA \\ ${ }^{2}$ Maryland Psychiatric Research Center, School of Medicine, University of Maryland, 55 Wade \\ Ave, Catonsville, MD 21228, USA \\ ${ }^{3}$ National Institute of Mental Health, Bldg 10, Room 1D80 Bethesda MD 20892, USA \\ ${ }^{4}$ Division of Biostatistics and Bioinformatics, School of Medicine, University of Maryland, 655 W. \\ Baltimore Street, Baltimore MD 21201, USA
}

\begin{abstract}
Cluster-wise statistical inference is the most widely used technique for functional magnetic resonance imaging (fMRI) data analyses. Cluster-wise statistical inference consists of two steps: i) primary thresholding that excludes less significant voxels by a pre-specified cut-off (e.g., $p<0.001$ ); and ii) cluster-wise thresholding that controls the family-wise error rate (FWER) caused by clusters consisting of false positive suprathreshold voxels. It has been well known that the selection of the primary threshold is critical because it determines both statistical power and false discovery rate. However, in most existing statistical packages, the primary threshold is selected based on prior knowledge (e.g., $p<0.001$ ) without taking into account the information in the data. In this manuscript, we propose a data-driven approach to objectively select the optimal primary threshold based on an empirical Bayes framework. We evaluate the proposed model using extensive simulation studies and an fMRI data example. The results show that our method can effectively increase statistical power while effectively controlling the false discovery rate.
\end{abstract}


Keywords: empirical Bayes, true positive proportion, true discovery proportion, optimal threshold

\section{Introduction}

Functional magnetic resonance imaging (fMRI) technique has become a popular tool for non-invasively studying circuit-level brain activity for more than two decades. Statistical analyses of neuroimaging data remain challenging due to their high-dimensionality and spatiotemporal dependence structure (Derado et al., 2010; Lindquist, 2020; Lindquist \& Mejia, 2015; Smith \& Nichols, 2018). Advanced statistical methods have been developed to solve the multiple comparison problem and successfully applied to many fMRI studies (Alberton et al., 2020; Chen et al., 2019; Nichols, 2012; Smith \& Nichols, 2009). Among these statistical methods, the cluster-wise inference remains the most commonly used tool for neuroimaging data analysis due to its relatively high sensitivity and low computational cost comparing to voxel-extent based thresholding methods (Friston et al., 1994; Woo et al., 2014). The performance and parameters of this procedure have been well discussed and studied (Eklund et al., 2018; Eklund et al., 2016; Flandin \& Friston, 2019; Y.-W. Hong et al., 2019).

A spatial point process perspective of cluster-wise inference. Cluster-wise inference consists of two steps: a primary thresholding step that applies a cut-off to all voxels and only keeps those supra-threshold voxels; and a cluster-extent- based thresholding step to avoid selecting false positive clusters under the null hypothesis (e.g., no activation). In this current study, we focus on the nonparametric inference method, due to its robustness although parametric methods (e.g., random field theory) may be more efficient when the assumptions are well met (Bennett, Wolford, et al., 2009; Eklund et al., 2015; Hayasaka \& Nichols, 2003; Nichols, 2012; Schwartzman \& Telschow, 2019). The high sensitivity of cluster-wise inference comparing to other multiple-testing correction methods (e.g., false discovery rate 
- FDR control) is built on the appropriate modeling of the stochastic spatial process for informative voxels. Specifically, the primary thresholding in step one binarizes all voxels to ones and zeros, and then the statistical inferences for the whole brain become a spatial point process in a 3D brain space where only supra-threshold voxels present as points (Kang et al., 2011). Under the null hypothesis, no brain area is associated with the external covariates, and the false positive points (voxels false-positively surviving the primary threshold) are assumed to follow a homogeneous spatial process. Under the alternative hypothesis, the identification of true events (voxels true-positively surviving the primary threshold) is a non-homogeneous/clustered process. Asymptotically, the combinatorial probability that true events connect (spatially adjacent) to each other and form a large cluster is much greater than the combinatorial probability of false positive voxels connecting to each other and forming a cluster with an equal or greater size. In that, we assume the noise is not related to any brain function or brain structure. However, the calculation of the asymptotic combinatorial probability is difficult because the relationship of the covariance between voxels and their geometric distance can be non-stationary and non-linear (Cressie, 2015). Fortunately, nonparametric test methods (e.g., permutation tests) can provide a good approximation of the asymptotic combinatorial probability of false positive voxels forming a cluster with a certain size since the empirical covariance between voxels is preserved in each permutation (Eklund et al., 2016; Nichols, 2012; Nichols \& Hayasaka, 2003). Nonparametric tests are also widely and successfully applied in other spatial point models, for example, SaTscan (Kulldorff, 2006) is used to monitor/detect a clustered spatial point incidences (disease outbreaks). Therefore, cluster-wise inference gains additional sensitivity by capitalizing on the patterns of positive voxels in a non-homogeneous process. However, the price for the additional power is the loss of local power (i.e., without voxel-level inference).

A key limitation for cluster-wise inference is its venerability to the poor selection of the voxel-level primary threshold. An overly-conservative threshold may lead to trivial clusters 
of connected true positive voxels and cause low statistical power. On the other hand, a liberal primary threshold (e.g., $p<0.01$ ) can generate massive false positive points in a smoothed brain space and thus can be connected and form false positive clusters (Woo et al., 2014). Both false positive clusters and low statistical power become major potential causes of the low reproducibility and replicability of fMRI findings (Lindquist, 2020).

Setting the primary threshold at $p<0.001$ is now standard for most studies, because it can generally effectively control false positive findings based on empirical studies (Bennett, Miller, et al., 2009; Woo et al., 2014). However, a pre-specified voxel-wise threshold may be sub-optimal because it does not account for several important factors of the data, including sample size, effect size, noise level, and the selection of statistical models, among many others. We illustrate the concept of data-driven optimal threshold selection by a toy example (Figure 1). We consider a simple scenario that all voxels in a brain image can be divided into two sets: those are truly associated with the covariate of interest and the rest. The test statistics of the two sets follow a non-null distribution and a null distribution, respectively. We argue that the optimal primary threshold should be selected based on the non-null and null distributions rather than a pre-specified threshold. For example, if the two distributions are well separated (large sample sizes or significantly strong signals), a more rigorous primary threshold (e.g., more stringent than 0.001) should be used, to suppress the false positive findings. In contrast, if the two distributions are less separable (but still separable, exhibiting small/moderate sample sizes and moderate/large effect sizes), a less conservative primary threshold (e.g., less conservative than 0.001 ) should be used to keep the false discovery rate at a low level while maintaining a maximal statistical power. Therefore, empirical distributions of null and non-null distribution can reflect the sample size, effect size, and noise level from the data and provide important guidance for primary threshold selection. 


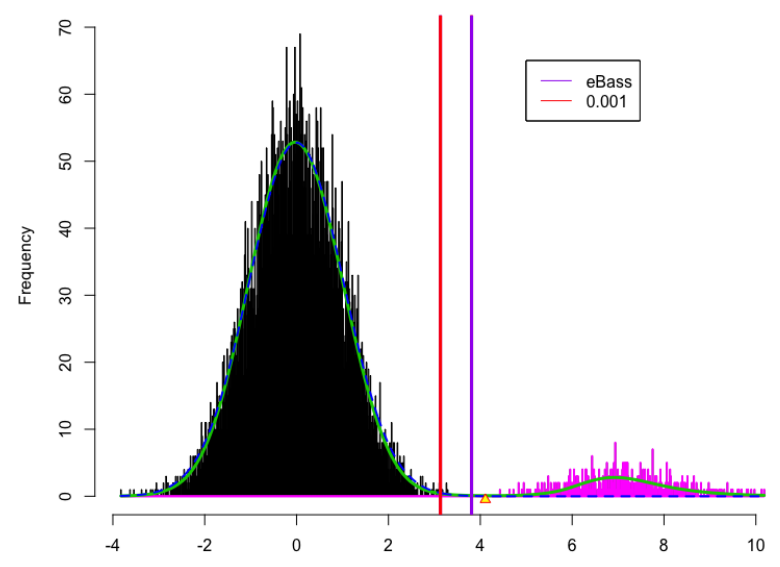

(a) Two distributions well separated

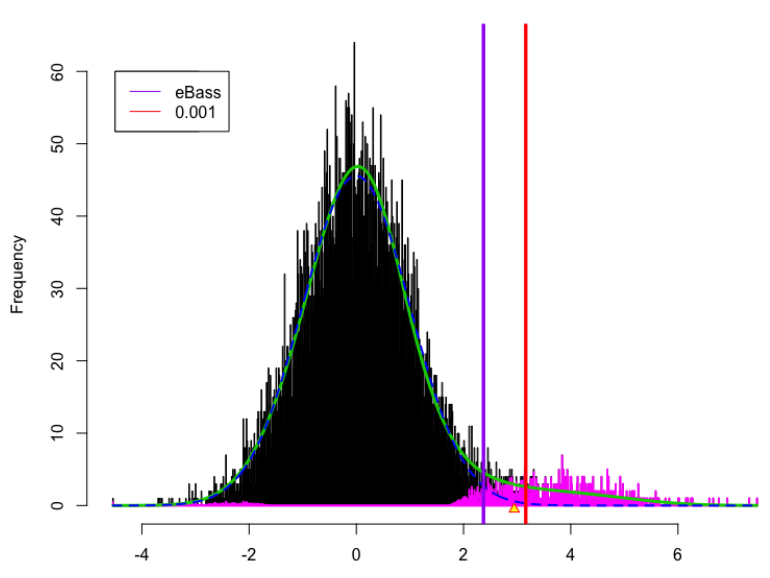

(b) Two distributions less separated

Figure 1: Non-null (in purple) and null (in black) distribution of test statistics from the (non-)event spatial points. The red vertical line marks down the corresponding z-score when the p-value is 0.001 on each graph. Yellow triangles on the $\mathrm{x}$-axis indicate threshold z-values for local fdr $\widehat{f d r}(z)=0.2$ if such cases exist. The null sub-density $\pi_{0} f_{0}$ is marked in a blue dashed line, and the mixture density is marked in a solid green line.

In the above example, we show that a data-driven primary threshold can maximize the statistical power to detect true positive clusters while effectively controlling the false discovery rate. However, the data-driven primary threshold selection procedure has not been fully developed. To fill this gap, we propose a new empirical Bayes Adaptive Threshold Selection (eBass) method to objectively select the optimal primary threshold selection based on the information from data. The eBass objective function aims to achieve a maximal statistical power while avoiding false positive voxels connected to be a false positive cluster. We develop new algorithms to implement the objective function and provide the corresponding theoretical properties. In this paper, we focus on the two-step cluster-wise fMRI inference with nonparametric statistical tests by providing a new primary threshold selection strategy. We note that alternative advanced statistical methods, including both nonparametric inference methods (e.g., threshold-free methods cluster enhancement - TFCE and pTFCE (Smith \& Nichols, 2009; Spisák et al., 2019)) and parametric inference (e.g., frequentist and 
Bayesian models (Benjamini \& Heller, 2007; Chen et al., 2019; Schwartzman \& Telschow, 2019)), can produce reliable and biologically meaningful results. Thus, eBass can become a complement to these commonly-used statistical approaches and enhance the widely used two-step cluster-wise inference.

The rest of this paper is organized as follows: 1) we introduce eBass method and algorithm in section $2 ; 2$ ) we perform extensive simulation analysis to fully assess the properties of eBass, in Section 3; 3) we apply eBass to an fMRI data analysis for schizophrenia research and conclude with discussions and future works.

\title{
2 Method
}

We consider the multiple comparison problem for all brain voxels in a spatially smooth $3 \mathrm{D}$ space. For example, the voxel-level brain activation to an external stimulus (task-induced fMRI) and seed voxel based brain connectivity map (resting-state fMRI). Let $v$ be an index for brain voxel and $v=1, \cdots, V$. We perform statistical inference on each voxel marginally (e.g., by a general linear model GLM), and conditionally (Bowman, 2005; Derado et al., 2010; Risk et al., 2016). Therefore, there are simultaneously $V$ hypothesis tests with their test statistics $\boldsymbol{Z}$ and corresponding $p$ values $\mathbf{P}$ :

\author{
Null Hypothesis : $H_{1}, H_{2}, H_{3}, \cdots H_{V}$ \\ Test Statistics : $z_{1}, z_{2}, z_{3}, \cdots z_{V}, p$-values $: p_{1}, p_{2}, p_{3}, \cdots p_{V}$
}

The commonly-used multiple testing correction methods ( Benjamini-Hochberg false discovery rate, or BH-FDR, correction) correct the multiplicity at the voxel level. The two-step cluster-wise inference aim to extract cluster-level findings and gain additional power (Nichols \& Hayasaka, 2003). Specifically, 
1. We consider the primary thresholding as a screening step. We first apply a predetermined threshold $\theta$ to binarize all voxels based on their $p$-values. Denote the indicator variable $\delta_{v}=I\left(p_{v}<\theta_{p}\right)$ (e.g., $\left.\theta_{p}<0.001\right)$ and $\Delta=\left\{\delta_{v}>0\right\}$, where $I$ is an indicator function. The binarization naturally leads to voxel-level false positive rate and sensitivity.

2. Perform permutation tests using the cluster extent as a test statistic to select a set of spatially adjacent supra-threshold voxels $\Delta$ as the resulting cluster while controlling the family-wise error rate (FWER). This step bears a resemblance to the commonly used spatial statistical models (e.g., SaTScan) that can competently handle an inhomogeneous spatial point process with clustered patterns (Waller \& Gotway, 2004). Thus, the final output is cluster-level findings (adjusting FWER).

We refer voxel-level false discovery rate as $\mathrm{vFDR}=\frac{\sum_{v}^{V} I\left(H_{v}=0\right)}{\sum_{v}^{V} I\left(\hat{H}_{v}=1\right)}$, and cluster-level familywise error as $\mathrm{cFWER}=\operatorname{Pr}($ at least one detected cluster is false positive). The two-step clusterwise inference controls cFWER instead of vFDR. Nevertheless, the vFDR can be used to evaluate the selected primary threshold in step one (screening).

It has been well-known that the choice of primary threshold is critical because i) an over conservative primary threshold can achieve a low voxel-level false discovery rate at the cost of low sensitivity (the cluster-level sensitivity is also low because true positive voxels are few to form clusters); ii) a liberal primary threshold can lead to detecting both true and false positive clusters (i.e., high cFWER). Either low sensitivity or high cFWER can lead to less replicable results because i) the probability is low to observe overlapped true findings across data sets with low sensitivity, and ii) the chance for false positive voxels reappearing in different data sets is small. Currently, the primary threshold of $p<0.001$ is well accepted by the research community, while $p<0.01$ is considered overly-liberal (Eklund et al., 2018). Here, we argue that a data-driven primary threshold may better balance the above trade-off 
than the pre-determined primary threshold.

We aim to select an optimal primary threshold to achieve maximal sensitivity (power) with a low false discovery rate at the voxel-level. In practice, however, neither voxel-level sensitivity nor FDR is known because the ground-truth is unavailable. We resort to an empirical Bayes framework for calculating the estimated voxel-level sensitivity and FDR.

\subsection{Empirical Bayes estimated voxel-level sensitivity and FDR}

The empirical Bayes framework has been developed to estimate the marginal distribution of the null and non-null test statistics for the multiple testing problem (Efron, 2012; Fan et al., 2012). In these models, the test statistics of the whole brain voxels follow a mixture distribution, $f(z)=\pi_{0} f_{0}(z)+\pi_{1} f_{1}(z)$, where $\pi_{0}, \pi_{1}\left(\pi_{1}=1-\pi_{0}\right)$ are the prior probabilities for a voxel belonging to the null and non-null components.

$$
\begin{array}{cc}
\pi_{0}=\operatorname{Pr}\left\{z_{v} \in \text { null }\right\}, & \text { null density } f_{0}\left(z_{v}\right) \\
\pi_{1}=\operatorname{Pr}\left\{z_{v} \in \text { non-null }\right\}, & \text { non-null density } f_{1}\left(z_{v}\right)
\end{array}
$$

The posterior probability of a voxel is from the null set given $\mathbf{z}$ is $\operatorname{Pr}\left\{z_{v} \in \operatorname{null} \mid \mathbf{z}\right\}=$ $\pi_{0} f_{0}(z) / f(z)$. A critical step of the empirical Bayes method is to estimate the mixture density $\hat{f}$. Fortunately, numerous efficient and robust numerical algorithms (e.g., MLE based and Poisson regression estimates) have been developed (Efron, 2014). The null density $\hat{f}_{0}$ can be estimated by maximum likelihood estimation and central matching method. The prior probability $\pi_{0}$ is estimated based no the estimated null distribution, and accordingly, $\hat{\pi}_{1}$ is $1-\hat{\pi}_{0}$. Generally, the empirical Bayes estimation method provides consistent and robust estimates for $\hat{\pi}_{0}, \hat{\pi}_{1}, \hat{f}_{0}, \hat{f}_{1} \mid \mathbf{Z}$ based on data $\mathbf{Z}$ (Efron, 2012; Petrone et al., 2014).

Given estimated $\operatorname{Pr}\left\{z_{v} \in\right.$ null $\}$ at each voxel, we can calculate the posterior sensitivity 
and FDR across all voxels at a cut-off $z_{\theta}$ by:

$$
\begin{aligned}
\text { Posterior Sensitivity: } & \widehat{\operatorname{TPR}}\left(z_{\theta}\right)=\int_{z_{\theta}}^{+\infty} \hat{f}_{1}(t) d t / \int_{-\infty}^{+\infty} \hat{f}_{1}(t) d t, \\
\text { Posterior FDR: } & \widehat{F D R}\left(z_{\theta}\right)=\hat{\pi}_{0} \int_{z_{\theta}}^{+\infty} \hat{f}_{0}(t) d t / \int_{z_{\theta}}^{+\infty} \hat{f}(t) d t
\end{aligned}
$$

and accordingly the true discovery rate (TDR) is

$$
\text { Posterior TDR } \widehat{\operatorname{TDR}}\left(z_{\theta}\right)=\hat{\pi}_{1} \int_{z_{\theta}}^{+\infty} \hat{f}_{1}(t) d t / \int_{z_{\theta}}^{+\infty} \hat{f}(t) d t \text {. }
$$

The $\widehat{T P R}\left(z_{\theta}\right)$ and $\widehat{T D R}\left(z_{\theta}\right)$ inherit the consistency property from empirical Bayes estimators (see Theorem 2 with detailed proofs in Appendix). Therefore, the empirical Bayes estimated $\widehat{T P R}\left(z_{\theta}\right)$ and $\widehat{T D R}\left(z_{\theta}\right)$ provide satisfactory surrogates for the true, yet unknown, sensitivity and FDR, which are required to determine the optimal threshold.

\subsection{Objective function for the optimal threshold}

Built on the EB posterior voxel-level sensitivity and FDR $\left(\widehat{T P R}\left(z_{\theta}\right)\right.$ and $\left.\widehat{F D R}\left(z_{\theta}\right)\right)$, we propose an objective function for optimal threshold selection. Specifically,

$$
\begin{gathered}
\underset{z_{\theta}}{\arg \max } \frac{\hat{\pi}_{1} \int_{z_{\theta}}^{+\infty} \hat{f}_{1}(t) d t}{\hat{\pi}_{1} \int_{-\infty}^{+\infty} \hat{f}_{1}(t) d t+\hat{\pi}_{1} \int_{z_{\theta}}^{+\infty} \hat{f}_{1}(t) d t+\left(1-\hat{\pi}_{1}\right) \int_{z_{\theta}}^{+\infty} \hat{f}_{0}(t) d t} \\
\text { subject to } \widehat{F W E} R_{\text {cluster }}\left(z_{\theta}\right) \leq \alpha,
\end{gathered}
$$

where the optimal cut-off $z_{\theta}$ is the estimand. The objective function (1) is the harmonic mean of empirical Bayes sensitivity and true discovery rate, and provides the optimal selection of $\hat{z}_{\theta}$ that maximizes the power with the family-wise error rate rate controlled under the level of $\alpha$ (see details of Theorem 1 in Appendix). The objective function is expressed by the 
empirical Bayes posterior parameters and functions $\hat{\pi}_{0}, \hat{\pi}_{1}, \hat{f}_{0}, \hat{f}_{1}$.

The cluster level family-wise error rate $\widehat{F W E} R_{\text {cluster }}\left(z_{\theta}\right)$ is determined by the number of estimated false positive voxels $\int_{z_{\theta}}^{+\infty} \hat{f}_{0}(t) d t$ and the cluster size cut-off. Specifically, we denote the estimated number of false positive voxels using a cut-off $z_{\theta}$ by $m_{f p}\left(z_{\theta}\right)=V \hat{\pi}_{0} \int_{z_{\theta}}^{+\infty} \hat{f}_{0}(t) d t$. Next, we implement the permutation procedure by relabelling to determine the clustersize cut-off with the FWER level $\alpha$ and primary threshold $z_{\theta}$. Let $K_{\alpha}\left(z_{\theta}\right)$ be the permutation determined cluster-size cut-off. When $z_{\theta}$ is liberal, $m_{f p}\left(z_{\theta}\right)$ tends to be a large number and thus is more likely to form large clusters composed by false positive voxels surpassing the cut-off $K_{\alpha}\left(z_{\theta}\right)$. In contrast, a stringent $z_{\theta}$ leads to a small $m_{f p}\left(z_{\theta}\right)$ prohibiting false positive voxels connecting into clusters greater than $K_{\alpha}\left(z_{\theta}\right)$. $\widehat{F W E R} R_{\text {cluster }}\left(z_{\theta}\right)=$ $\operatorname{Pr}\left(\operatorname{Sup}\left\{\mu\left(m_{f p}\left(z_{\theta}\right)\right)\right\} \geq K_{\alpha}\right)$, where $\mu$ is the cardinality measure of any set of contiguous voxels formed by $m_{f p}\left(z_{\theta}\right)$ in the brain space. Then, we define the search domain for $\hat{z}_{\theta}$ by $\Omega_{\alpha}=\left\{z_{\theta}: \operatorname{Pr}\left(\operatorname{Sup}\left\{\mu\left(m_{f p}\left(z_{\theta}\right)\right)\right\} \geq K_{\alpha}\right)<\alpha\right\}$. We provide the computational details to calculate $\operatorname{Sup}\left\{\mu\left(m_{f p}\left(z_{\theta}\right)\right)\right\}$ estimate $\Omega$ in the Appendix. Next, we optimize $\hat{z}_{\theta}$ on the support $\Omega$.

We summarize the computational procedure of eBass in three steps:

Step 1: Calculate $\widehat{T P R}\left(z_{\theta}\right), \widehat{F D R}\left(z_{\theta}\right)$ with the empirical Bayes estimated $\hat{\pi}_{0}, \hat{\pi}_{1}, \hat{f}_{0}, \hat{f}_{1}$, and the objective function as

$$
h\left(z_{\theta}, \hat{\pi}_{0}, \hat{\pi}_{1}, \hat{f}_{0}, \hat{f}_{1}\right)=\frac{\hat{\pi}_{1} \int_{z_{\theta}}^{+\infty} \hat{f}_{1}(t) d t}{\hat{\pi}_{1} \int_{-\infty}^{+\infty} \hat{f}_{1}(t) d t+\hat{\pi}_{1} \int_{z_{\theta}}^{+\infty} \hat{f}_{1}(t) d t+\left(1-\hat{\pi}_{1}\right) \int_{z_{\theta}}^{+\infty} \hat{f}_{0}(t) d t}
$$

Step 2: Identify the support $\Omega_{\alpha}$ which guarantees $\widehat{F W E} R_{\text {cluster }} \leq \alpha$ (see Appendix).

Step 3: Optimize the $z_{\theta}$ by $\arg \max h\left(\hat{\pi}_{0}, \hat{\pi}_{1}, \hat{f}_{0}, \hat{f}_{1}\right)$ subject to constraint in Step 2, under the $z_{\theta} \in \Omega_{\alpha}$ regularity condition for Cost-Sensitive Classification algorithm (Eban et al., 2017). According to the algorithm, the objective function $h(\cdot)$ reduces to a weighted classification problem, where the errors are approximated by cost-sensitive binary classification algorithms (e.g., logistic regression) with asymmetric costs. 
The optimization of (1) yields the optimal primary threshold $\hat{z}_{\theta}$, which maximizes the

sensitivity with $\widehat{F W E} R_{\text {cluster }} \leq \alpha$. Note that $\hat{z}_{\theta}$ is objectively selected by fully leveraging the information from the empirical data. In practice, the sample size, effect size, noise level, spatial dependence across all voxels of the empirical data set can influence the separability of the null and non-null distribution, and thus the optimal primary threshold should be updated to accommodate these factors. The empirical Bayes estimated FDR and sensitivity determines our objective function (1), and bridges the data information and parameter optimization. The data-driven primary threshold selection method eBass automatically maximizes the empirical Bayes sensitivity while controlling voxel-level FDR and then cluster-level FWER. Therefore, eBass primary threshold can outperform the pre-specified primary thresholds in many scenarios (see simulation and data example results).

In summary, we provide a data-driven optimal primary threshold selection step via an empirical Bayes framework. The selection of the primary threshold is more flexible because eBass optimizes the primary threshold by maximizing the sensitivity while rigorously controlling the cluster-wise false positive error rate. We provide theoretical proof details for the optimality (Theorem 1 in Appendix) and consistency (Theorem 2 in Appendix). In the following simulation analysis and real data example, we demonstrate that eBass can improve the statistical power without losing the rigor of FWER.

\section{Simulation}

\subsection{Data Description}

In the simulation study, we evaluate the performance of eBass and compare it to the existing methods. We first simulate 2D image data for multiple subjects. The number of voxels in each image is $V=100 \times 100=10,000$, and thus the number of simultaneous tests is 10,000. We assume that most voxels are from the null set, whereas two squared areas 
$\left(N_{0}=21 \times 21+6 \times 6=477\right.$ voxels $)$ are from the non-null, see Fig $2 \mathrm{~b}$. We apply a commonly-used two-group (i.e., cases vs. controls) scenario, which can be easily extended to the regression setting. First, let voxels from the null set follow a normal distribution $N(0,1)$ for both cases and controls. Within the two squared areas, the non-null voxels of the cases follow a normal distribution $N\left(\mu_{k}, 1\right)(k=1,2$ for the two areas), whereas the voxels of the controls follow a $N(0,1)$ distribution. The signal-to-noise ratio (SNR) as the reciprocal of the coefficient of variation, $S N R=\mu_{k} / \sigma$, the $\sigma=1$ allows the difference of group means to be the true positive effect size (ES) which is equivalent to Cohen's d. A higher SNR can lead to higher sensitivity and a lower FDR, and vice versa. We further smooth each image with a Gaussian filter, using a full width at half maximum (FWHM) equivalent to 8mm. The voxels in the smoothed image are correlated like the real fMRI data. We further let the number of subjects per group be 30,60, and 100. For each setting, we simulate 100 data sets.

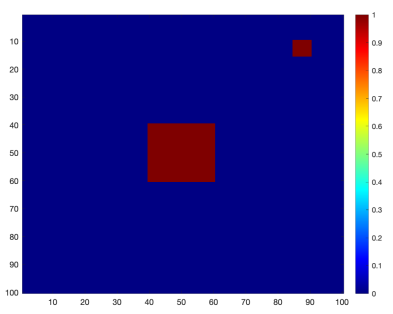

(a) Underlying truth

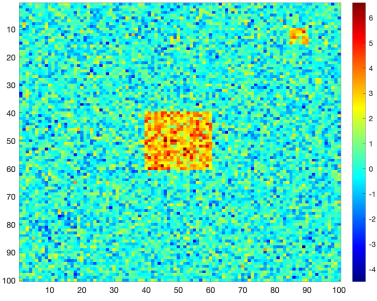

(b) Original graph

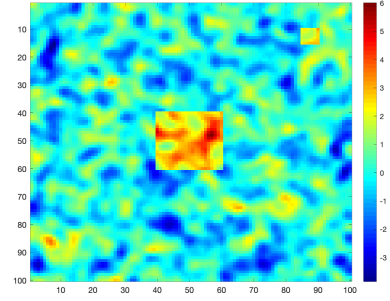

(c) Smoothed graph with $\mathrm{FWHM}=8 \mathrm{~mm}$

Figure 2: (a) is the graph of the underlying truth. Red squares represent the activated regions. (b) shows one original graph with sample size 30 per arm, an effect size set to 0.8. Non-activated voxels in blue region of (a) generated that follow $N(0,1)$ and activated voxels in red regions of (a) follow $N(0.8,1)$. (c) was the original graph in (b) smoothed with a Gaussian kernel of FWHM=8mm. 


\subsection{Data analysis}

For each data set, we perform the two-step cluster-wise inference. We determine the primary threshold using eBass as well as a variety of popular methods, including BH-FDR correction, $p<0.001$, and $p<0.01$. We evaluate the performance of these methods in terms of voxelwise sensitivity (Sensitivity) and FDR (vFDR), together with cluster-wise FWER (cFWER) by comparing the resulting clusters selected by the cluster-wise inference to the two true squares. Note that ultimately, the voxel-wise sensitivity and FDR are not applicable in cluster-wise inference, we yet provide them in order to evaluate the quality of controls in the voxel-level primary thresholding step.

\subsection{Results}

We summarize simulation results in Table 1 . We first compare the results when the effect size is medium $(\mathrm{ES}=0.6)$. For the sample size of 30 cases vs. 30 controls, the study is underpowered and the test statistics from the true positive voxels are mixed with false positive voxels (Cremers et al., 2017; Lindquist et al., 2008). Therefore, only a few voxels can survive the corrected primary threshold and form a cluster with a size greater than the step two cluster-level threshold. The vFDRs for all methods are well controlled. The Sensitivity of eBass is $137 \%$ higher that $p<0.001$ threshold and $388 \%$ higher than BH-FDR correction. Although the well-controlled vFDR prohibits false positive findings, the overall low sensitivity can also lead to a low replicability because true positive findings are rarely overlapped across data sets.

When the sample size is increased to 60 subjects per arm, the statistical power increases to above $80 \%$ at each voxel. The vFDRs of eBass, BH-FDR correction, and $p<0.001$ primary threshold are around 0.005 , while vFDR of threshold $p<0.01$ is close to 0.013 . Note that our eBass primary threshold varies across repeatedly simulated data sets, and 
automatically adapt to the characteristics of the data. In that, our average vFDR is about the same as $p<0.001$ and BH-FDR, but has a significantly increased Sensitivity. Since the sample size of 60 vs. 60 and $\mathrm{ES}=0.6$ is common in fMRI studies, these results provide practical guidance for optimal primary threshold selection for cluster-wise inference.

Last, for sample size of 100 vs. 100, the test statistics of voxels from the null set are clearly apart from those from the non-null set, which leads to generally increased Sensitivity in all methods. The eBass method has slightly higher Sensitivity than that of $p<0.001$ with better controlled vFDR because of its adaptive optimal threshold selection. The cFWER for all methods are well-controlled.

The results of larger effect sizes $(\mathrm{ES}=0.8$ and 1$)$ follow a similar pattern as above (see Table 1). When the sample size is small (i.e., 30 cases vs. 30 controls), eBass significantly improves Sensitivity while keeping the vFDR at a very low level. At effect size 0.8 and the sample size is medium to large (i.e., 60, 100 subjects per arm), adaptive threshold selection methods eBass and BH-FDR correction performed slightly superior to the fixed primary threshold $p<0.001$. When the effect size reaches to 1.0 , there is not much difference in Sensitivity among all methods especially when sample size is very large (100 subjects per arm). All methods control the vFDR well when effect sizes increase. Since we focus on cluster-wise inference and cluster-level FWER, we only compare eBass with existing clusterwise inference methods. In the appendix, we show the comparison results of TFCE and cluster-wise inference methods including eBass by assuming all voxels in significant clusters as positive.

In summary, eBass shows advantageous performance in improving the sensitivity while controlling the voxel-wise FDR, specially when the sample size and effect size is small to medium. With increased sample size and effect size, most of the widely used thresholding methods tend to have a good performance with similar primary thresholds. 
bioRxiv preprint doi: https://doi.org/10.1101/2020.10.03.324962; this version posted October 27, 2020. The copyright holder for this preprint (which was not certified by peer review) is the author/funder, who has granted bioRxiv a license to display the preprint in perpetuity. It is made available under aCC-BY-NC 4.0 International license.

\begin{tabular}{|c|c|c|c|c|c|}
\hline & & "eBass & "BH-FDR & $\bar{p} p<0.001$ & $p<0.01$ \\
\hline \multicolumn{6}{|c|}{ Effect Size $=0.6$} \\
\hline & Threshold & $0.0027(\mathrm{Q} 1) / 0.0051(\mathrm{Q} 2) / 0.0147(\mathrm{Q} 3)$ & $0.0002(\mathrm{Q} 1) / 0.0009(\mathrm{Q} 2) / 0.0017(\mathrm{Q} 3)$ & 0.001 & 0.01 \\
\hline Sample size & Sensitivity & $0.1932 \pm 0.0934$ & $0.0396 \pm 0.0325$ & $0.0816 \pm 0.0344$ & $0.2263 \pm 0.081$ \\
\hline \multirow[t]{2}{*}{30 per arm } & vFDR & $0.024 \pm 0.0653$ & 0 & $0.0117 \pm 0.0469$ & 0 \\
\hline & cFWER & $5.6 \%$ & 0 & $6.3 \%$ & 0 \\
\hline & Threshold & $0.0012(\mathrm{Q} 1) / 0.0027(\mathrm{Q} 2) / 0.0056(\mathrm{Q} 3)$ & $0.0008(\mathrm{Q} 1) / 0.0009(\mathrm{Q} 2) / 0.0013(\mathrm{Q} 3)$ & 0.001 & 0.01 \\
\hline Sample size & Sensitivity & $0.5039 \pm 0.1237$ & $0.3731 \pm 0.1481$ & $0.3777 \pm 0.1075$ & $0.6763 \pm 0.1164$ \\
\hline \multirow[t]{2}{*}{60 per arm } & vFDR & $0.0067 \pm 0.0155$ & $0.0050 \pm 0.0154$ & $0.0046 \pm 0.0144$ & $0.0127 \pm 0.0256$ \\
\hline & cFWER & $5 \%$ & $10 \%$ & $10 \%$ & $10 \%$ \\
\hline & Threshold & $0.0009(\mathrm{Q} 1) / 0.0020(\mathrm{Q} 2) / 0.0032(\mathrm{Q} 3)$ & $0.0020(\mathrm{Q} 1) / 0.0021(\mathrm{Q} 2) / 0.0022(\mathrm{Q} 3)$ & 0.001 & 0.01 \\
\hline Sample size & Sensitivity & $0.8292 \pm 0.0749$ & $0.8460 \pm 0.0767$ & $0.7850 \pm 0.081$ & $0.9378 \pm 0.0422$ \\
\hline \multirow{2}{*}{100 per arm } & vFDR & 0 & $0.0019 \pm 0.0049$ & $0.0001 \pm 0.0006$ & 0 \\
\hline & cFWER & 0 & 0 & 0 & 0 \\
\hline
\end{tabular}

Effect Size $=0.8$

\begin{tabular}{|c|c|c|c|c|c|}
\hline & Threshold & $0.0073(\mathrm{Q} 1) / 0.0097(\mathrm{Q} 2) / 0.0106(\mathrm{Q} 3)$ & $0.0006(\mathrm{Q} 1) / 0.0007(\mathrm{Q} 2) / 0.0009(\mathrm{Q} 3)$ & 0.001 & 0.01 \\
\hline Sample size & Sensitivity & $0.6012 \pm 0.0886$ & $0.2881 \pm 0.1141$ & $0.3146 \pm 0.0848$ & $0.6109 \pm 0.0914$ \\
\hline \multirow[t]{2}{*}{30 per arm } & vFDR & $0.004 \pm 0.01$ & $0.0045 \pm 0.0201$ & 0 & $0.0039 \pm 0.0096$ \\
\hline & cFWER & 0 & $5 \%$ & 0 & 0 \\
\hline & Threshold & $0.0034(\mathrm{Q} 1) / 0.0050(\mathrm{Q} 2) / 0.0055(\mathrm{Q} 3)$ & $0.0021(\mathrm{Q} 1) / 0.0023(\mathrm{Q} 2) / 0.0023(\mathrm{Q} 3)$ & 0.001 & 0.01 \\
\hline Sample size & Sensitivity & $0.9438 \pm 0.0396$ & $0.9072 \pm 0.0552$ & $0.8559 \pm 0.0605$ & $0.9624 \pm 0.0376$ \\
\hline \multirow[t]{2}{*}{60 per arm } & vFDR & $0.0109 \pm 0.0252$ & $0.0052 \pm 0.0156$ & $0.0049 \pm 0.0129$ & $0.0119 \pm 0.0263$ \\
\hline & cFWER & $15 \%$ & $10 \%$ & $15 \%$ & $10 \%$ \\
\hline & Threshold & $0.0011(\mathrm{Q} 1) / 0.0016(\mathrm{Q} 2) / 0.0020(\mathrm{Q} 3)$ & $0.0024(\mathrm{Q} 1) / 0.0024(\mathrm{Q} 2) / 0.0025(\mathrm{Q} 3)$ & 0.001 & 0.01 \\
\hline Sample size & Sensitivity & $0.9945 \pm 0.0095$ & $0.9955 \pm 0.0087$ & $0.9897 \pm 0.0145$ & $0.9988 \pm 0.0030$ \\
\hline \multirow[t]{2}{*}{100 per arm } & vFDR & $0.0036 \pm 0.0095$ & $0.0023 \pm 0.0073$ & $0.0025 \pm 0.0078$ & $0.0064 \pm 0.0147$ \\
\hline & cFWER & $10 \%$ & $5 \%$ & $10 \%$ & $5 \%$ \\
\hline \multicolumn{6}{|c|}{ Effect Size $=1.0$} \\
\hline \multirow{4}{*}{$\begin{array}{l}\text { Sample size } \\
30 \text { per arm }\end{array}$} & Threshold & 0.0093(Q1)/0.0119(Q2)/0.0154(Q3) & $0.0014(\mathrm{Q} 1) / 0.0016(\mathrm{Q} 2) / 0.0018(\mathrm{Q} 3)$ & 0.001 & 0.01 \\
\hline & Sensitivity & $0.8539 \pm 0.0522$ & $0.6306 \pm 0.1122$ & $0.5781 \pm 0.0982$ & $0.8432 \pm 0.0526$ \\
\hline & vFDR & $0.0093 \pm 0.0189$ & $0.0045 \pm 0.0138$ & $0.0026 \pm 0.0115$ & $0.0057 \pm 0.0168$ \\
\hline & cFWER & $5 \%$ & $10 \%$ & $5 \%$ & $5 \%$ \\
\hline \multirow{4}{*}{$\begin{array}{l}\text { Sample size } \\
60 \text { per arm }\end{array}$} & Threshold & $0.0015(\mathrm{Q} 1) / 0.0022(\mathrm{Q} 2) / 0.0031(\mathrm{Q} 3)$ & $0.0023(\mathrm{Q} 1) / 0.0024(\mathrm{Q} 2) / 0.0025(\mathrm{Q} 3)$ & 0.001 & 0.01 \\
\hline & Sensitivity & $0.9851 \pm 0.0154$ & $0.9842 \pm 0.0191$ & $0.9727 \pm 0.0281$ & $0.9951 \pm 0.0087$ \\
\hline & vFDR & $0.0003 \pm 0.0014$ & $0.0002 \pm 0.0006$ & $0.0027 \pm 0.0085$ & $0.0097 \pm 0.0177$ \\
\hline & cFWER & 0 & 0 & $10 \%$ & $5 \%$ \\
\hline \multirow{4}{*}{$\begin{array}{l}\text { Sample size } \\
100 \text { per arm }\end{array}$} & Threshold & $0.0004(\mathrm{Q} 1) / 0.0006(\mathrm{Q} 2) / 0.0008(\mathrm{Q} 3)$ & $0.0024(\mathrm{Q} 1) / 0.0024(\mathrm{Q} 2) / 0.0025(\mathrm{Q} 3)$ & 0.001 & 0.01 \\
\hline & Sensitivity & 1 & 1 & 1 & 1 \\
\hline & vFDR & 0 & $0.0015 \pm 0.0034$ & 0 & $0.0067 \pm 0.0105$ \\
\hline & cFWER & 0 & 0 & 0 & 0 \\
\hline
\end{tabular}

Table 1. Simulation result for original images of $\mathrm{ES}=0.6,0.8$ and 1.0, sample size 30, 60 and 100 per arm smoothed with FWHM=8mm 


\section{Data Example}

\subsection{Data Acquisition}

Resting-state fMRI (Rs-fMRI) data were collected from 96 schizophrenia patients (SZs) at the University of Maryland Center for Brain Imaging Research. The average age of the SZ cohort is $35.9 \pm 13.2,28$ of the participants are females. A Siemens 3T TRIO MRI (Erlangen, Germany) system equipped with a 32-channel phase array head coil was used to collect the resting-state $T 2^{*}$-weighted images with the following parameters: $T R=2 \mathrm{~s}, \mathrm{TE}=30 \mathrm{~ms}$, flip angle $=90^{\circ}, \mathrm{FOV}=248 \mathrm{~mm}, 128 \times 128$ matrix, $1.94 \times 1.94$ in-plane resolution, $4 \mathrm{~mm}$ slice thickness, 37 axial slices, 444 volumes). During the scan, participants were asked to keep their eyes closed and relax.

\subsection{Data Preprocessing}

Pre-processing of the rs-fMRI data was performed using the Data Processing \& Analysis for (resting-state) Brain Imaging (DPABI) toolbox (Yan et al., 2016). The first ten-time frames were removed to allow for signal stabilization. Raw data underwent motion correction to the first image, slice-timing correction to the middle slice, and normalization to MNI space. To ensure that spurious motion and physiological artifacts did not drive observed effects in our statistical analyses, resting data also underwent regression of 6-motion parameters and their derivatives (12 total motion estimates) and physiological (white matter and cerebrospinal fluid) signals prior to spatial smoothing with an 8mm FWHM Gaussian kernel. Framewise displacement was calculated for each image; this measure differentiates head realignment parameters across frames and generates a 6-dimensional times series that represents instantaneous head motion (Power et al., 2012). All individuals in the current analysis had mean framewise displacement $\leq 0.25$ to better control for potential confounding effects of motion and motion artifacts on the rs-fMRI signal. 


\subsection{Data Analysis}

We aimed to examine the association of functional connectivity of the default mode network with smoking status. The seed voxel method was used by placing a $10 \mathrm{~mm}$ spherical seed centered on the precuneus/posterior cingulate centered (PCC). The correlations were calculated between the rest of voxels and the seed, and then normalized by the Fisher's Z transformation. The two-step cluster-wise inference was conducted to identify smokingrelated voxel-clusters.

In step one, voxel-wise regression was performed with the factor of interest (e.g., smoking status) on 215,348 nonzero voxels while adjusting for age and gender (Hare et al., 2020). We first applied the eBass approach and selected the optimal primary threshold by balancing the empirical Bayes $\widehat{T P R}, \widehat{F W E R}$ in the objective function (1). The eBass optimal threshold was 0.0018 , which was slightly more liberal than $p<0.001$ (see Figure 3). Next, step two permutation tests were performed based on the primary threshold to identify the significant clusters by controlling FWER $<0.05$.

We compared the eBass primary threshold with the popular primary threshold values including $p<0.001$ and $p<0.01$. We demonstrate the results in Figure 3 as well. All the significant regions were marked in red. The eBass primary threshold based cluster-wise inference yielded significant clusters at left cuneus, superior occipital gyrus and superior parietal lobule, and slightly expanded to right precuneus and cuneus.

In contrast, no significant cluster was detected by the primary threshold of $p<0.001$ while over-sized clusters were detected by the primary threshold $p<0.01$ expanding multiple anatomical brain gyri/sulci (e.g., extended to right calcarine gyrus and multiple contiguous regions on right brain, see Fig3). The clusters based on eBass primary threshold indicate increases in activation at the marked regions, which are highly overlapped with previous findings (Fedota \& Stein, 2015; E. Hong et al., 2011). Therefore, the data-driven eBass primary threshold is more flexible and provides a better balance between the sensitivity and 
false discovery rate, which should lead to greater replicability.

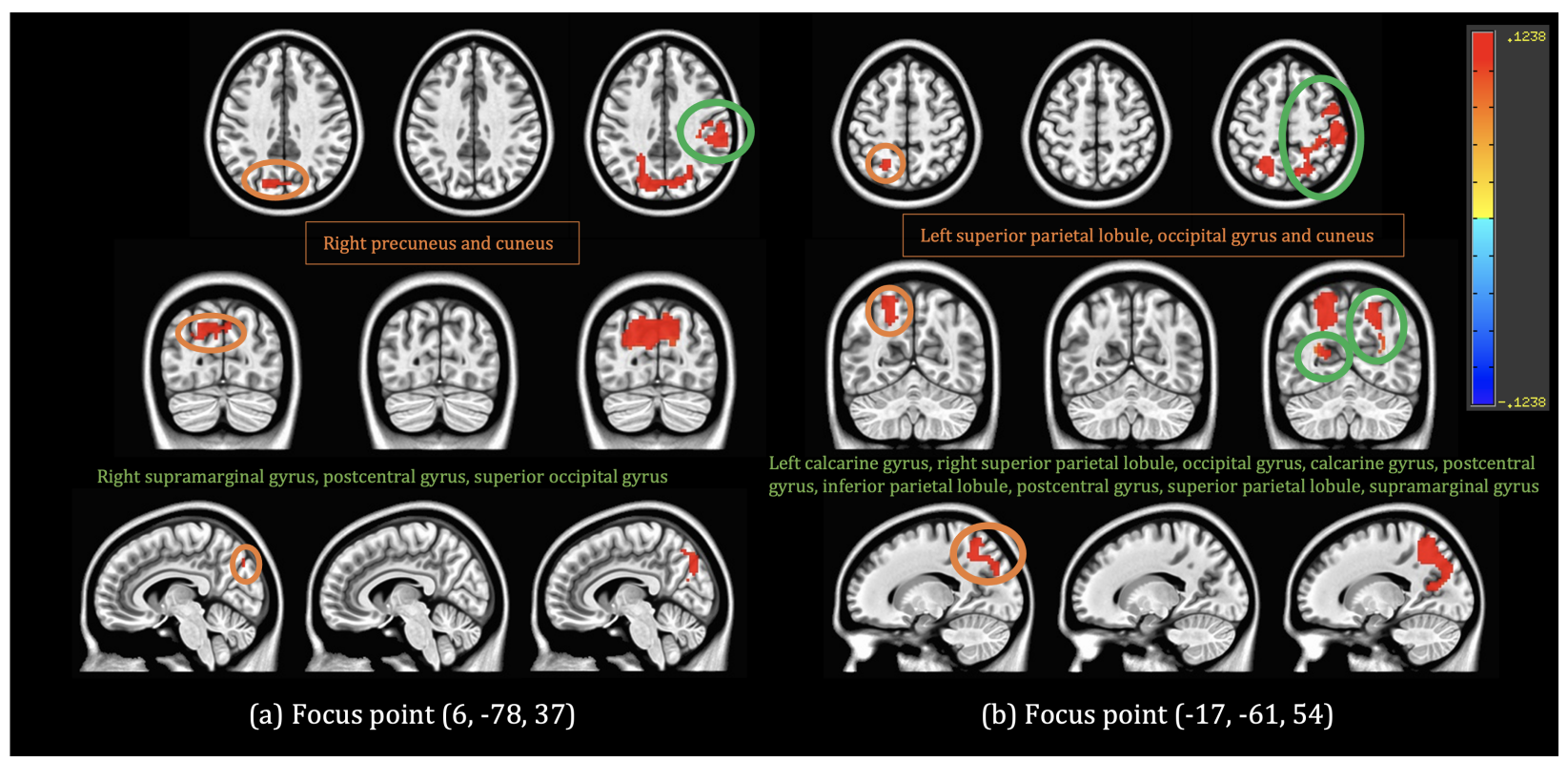

Figure 3: Two groups (in (a), (b)) of significant regions given by Primary thresholds of eBass (column 1), $p<0.001$ (column 2), and $p<0.01$ (column 3): axial (row 1), coronal (row 2) and sagittal (row 3) view. (a) focus point is located at $(6,-78,37$ ) on the right brain, (b) focus point is located at $(-17,-61,54)$ on the left brain. Regions overlap with existing schizophrenia vs nicotine studies were circled by orange line and listed under the first row in the solid frame. The additional regions detected by $p<0.01$ were circled by green line and listed under the second row without any frame.

\section{Discussion}

We have developed a data-driven primary threshold selection method for the two-step clusterwise fMRI inference. The multiple comparison problem has been at the heart of neuroimaging data analysis, because it can determine the validity of findings. In practice, true signals in neuroimaging data are often mixed with various sources of noise, and the statistical inference models are sensitive to the noise. Therefore, a small erroneous shift from the optimal decision-making threshold can cause a significant loss of statistical power or uncontrolled false positive findings. However, the primary threshold has been conventionally selected based on empirical analysis and experience, which may not provide the optimal threshold 
for the target neuroimaging data. To address this need, we propose an empirical Bayes method to calculate estimated sensitivity and false discovery rate and thus facilitate the optimization of selecting the primary threshold for cluster-wise inference.

Built on the successful development of the empirical Bayes approach in the field of highdimensional statistics, eBass enjoys several advantageous theoretical properties, regarding the estimation robustness and consistency (Efron, 2014; Schwartzman et al., 2009). The eBass threshold provides a reliable cut-off to binarize voxels in the $3 \mathrm{D}$ brain space into a point process (Kang et al., 2011). The step two inference (i.e., permutation tests) is also sensitive to the noise level of the point process. When the sensitivity level is low (a stringent threshold), true positive points are unlikely to be spatially adjacent and form a non-trivial cluster resulting in a reduced ability to detect no significant clusters. When a large proportion of false positive points are present in the point process, the false positive points tend to be spatially connected due to the spatial smoothness of the neuroimaging data, leading to cluster-wise false positive findings. For these reasons, we often find it challenging to produce replicable findings in neuroimaging studies (Lindquist, 2020).

Our simulation and data example results concur with the previous findings that the empirical primary threshold $(p<0.001)$ is a good option, especially when no information from the data is available. In general, the primary threshold $(p<0.001)$ can adequately control the false positive findings, which is analogous to the traditional cut of $p<0.05$ in univariate statistical inference (Eklund et al., 2018). In practice, we find that the data-driven eBass threshold often varies around the primary threshold $p<0.001$, in many applications. Nevertheless, the eBass primary threshold is objectively selected based on the data, and can thus improve sensitivity in many scenarios (e.g., data sets with smaller sample sizes and small-medium effect sizes). Therefore, we consider the eBass primary threshold to be a good complement to the existing methods for cluster-wise inference. We also note that the eBass is built on the estimation of the two-component mixture model. When the empirical Bayes 
approach cannot estimate the two components well, we resort to the $p<0.001$ primary threshold for cluster-wise inference or TFCE as potential solutions.

The eBass method is compatible with all voxel-level statistical inference because the marginal distribution of test statistics is often robust (Chen et al., 2019). The more accurate voxel-level statistical inference can lead to more separable null and non-null distributions and thus more accurate cluster-wise inference results via the eBass primary threshold.

In summary, the eBass provides a data-driven and automatically optimized primary threshold for the two-step cluster-wise fMRI inference. Since the computation is efficient, eBass can be conveniently implemented and compatible with most existing software platforms.

\section{References}

Alberton, B. A., Nichols, T., Gamba, H., \& Winkler, A. (2020). Multiple testing correction overcontrasts for brain imaging. NeuroImage, 116760.

Benjamini, Y., \& Heller, R. (2007). False discovery rates for spatial signals. Journal of the American Statistical Association, 102(480), 1272-1281.

Bennett, C., Miller, M., \& Wolford, G. (2009). Neural correlates of interspecies perspective taking in the post-mortem atlantic salmon: An argument for multiple comparisons correction. Neuroimage, 47 (Suppl 1), S125.

Bennett, C., Wolford, G., \& Miller, M. (2009). The principled control of false positives in neuroimaging. Social cognitive and affective neuroscience, 4(4), 417-422.

Bowman, F. D. (2005). Spatio-temporal modeling of localized brain activity. Biostatistics, $6(4), 558-575$. 
Chen, G., Xiao, Y., Taylor, P., Rajendra, J., Riggins, T., Geng, F., Redcay, E., \& Cox, R. (2019). Handling multiplicity in neuroimaging through bayesian lenses with multilevel modeling. Neuroinformatics, 17(4), 515-545.

Cremers, H., Wager, T., \& Yarkoni, T. (2017). The relation between statistical power and inference in fmri. PloS one, 12(11), e0184923.

Cressie, N. (2015). Statistics for spatial data. John Wiley \& Sons.

Derado, G., Bowman, D., \& Kilts, C. (2010). Modeling the spatial and temporal dependence in fmri data. Biometrics, 66(3), 949-957.

Eban, E., Schain, M., Mackey, A., Gordon, A., Rifkin, R., \& Elidan, G. (2017). Scalable learning of non-decomposable objectives, In Artificial intelligence and statistics.

Efron, B. (2012). Large-scale inference: Empirical bayes methods for estimation, testing, and prediction (Vol. 1). Cambridge University Press.

Efron, B. (2014). Two modeling strategies for empirical bayes estimation. Statistical science: a review journal of the Institute of Mathematical Statistics, 29(2), 285.

Eklund, A., Knutsson, H., \& Nichols, T. (2018). Cluster failure revisited: Impact of first level design and physiological noise on cluster false positive rates. Human brain mapping, $40(7), 2017-2032$.

Eklund, A., Nichols, T., \& Knutsson, H. (2015). Can parametric statistical methods be trusted for fmri based group studies? arXiv preprint arXiv:1511.01863.

Eklund, A., Nichols, T., \& Knutsson, H. (2016). Cluster failure: Why fmri inferences for spatial extent have inflated false-positive rates. Proceedings of the national academy of sciences, 113(28), 7900-7905.

Fan, J., Han, X., \& Gu, W. (2012). Estimating false discovery proportion under arbitrary covariance dependence. Journal of the American Statistical Association, 107(499), 1019-1035. 
Fedota, J., \& Stein, E. (2015). Resting-state functional connectivity and nicotine addiction: Prospects for biomarker development. Annals of the New York Academy of Sciences, $1349(1), 64$.

Flandin, G., \& Friston, K. (2019). Analysis of family-wise error rates in statistical parametric mapping using random field theory. Human brain mapping, 40(7), 2052-2054.

Friston, K., Worsley, K., Frackowiak, R. S., Mazziotta, J., \& Evans, A. (1994). Assessing the significance of focal activations using their spatial extent. Human brain mapping, $1(3), 210-220$.

Hare, S., Chiappelli, J., Savransky, A., Adhikari, B., Wisner, K., Kvarta, M., Goldwaser, E., Du, X., Chen, S., Rowland, L., Et al. (2020). The role of hippocampal functional connectivity on multisystem subclinical abnormalities in schizophrenia. Psychosomatic medicine, 82(6), 623-630.

Hayasaka, S., \& Nichols, T. (2003). Validating cluster size inference: Random field and permutation methods. Neuroimage, 20(4), 2343-2356.

Hong, E., Schroeder, M., Ross, T., Buchholz, B., Salmeron, B. J., Wonodi, I., Thaker, G., \& Stein, E. (2011). Nicotine enhances but does not normalize visual sustained attention and the associated brain network in schizophrenia. Schizophrenia bulletin, 37(2), 416425.

Hong, Y.-W., Yoo, Y., Han, J., Wager, T. D., \& Woo, C.-W. (2019). False-positive neuroimaging: Undisclosed flexibility in testing spatial hypotheses allows presenting anything as a replicated finding. NeuroImage, 195, 384-395.

Kang, J., Johnson, T., Nichols, T., \& Wager, T. (2011). Meta analysis of functional neuroimaging data via bayesian spatial point processes. Journal of the American Statistical Association, 106(493), 124-134.

Kulldorff, M. (2006). Satscantm user guide for version 7.0. SaTScanTM. Accessed on August, $13,2007$. 
Lindquist, M. (2020). Neuroimaging results altered by varying analysis pipelines. Nature Publishing Group.

Lindquist, M. Et al. (2008). The statistical analysis of fmri data. Statistical science, 23(4), 439-464.

Lindquist, M., \& Mejia, A. (2015). Zen and the art of multiple comparisons. Psychosomatic medicine, $77(2), 114$.

Nichols, T. (2012). Multiple testing corrections, nonparametric methods, and random field theory. Neuroimage, 62(2), 811-815.

Nichols, T., \& Hayasaka, S. (2003). Controlling the familywise error rate in functional neuroimaging: A comparative review. Statistical methods in medical research, 12(5), 419446.

Parambath, S. P., Usunier, N., \& Grandvalet, Y. (2014). Optimizing f-measures by costsensitive classification, In Advances in neural information processing systems.

Petrone, S., Rousseau, J., \& Scricciolo, C. (2014). Bayes and empirical bayes: Do they merge? Biometrika, $101(2)$, 285-302.

Power, J. D., Barnes, K. A., Snyder, A. Z., Schlaggar, B. L., \& Petersen, S. E. (2012). Spurious but systematic correlations in functional connectivity mri networks arise from subject motion. Neuroimage, 59(3), 2142-2154.

Risk, B., Matteson, D., Spreng, N., \& Ruppert, D. (2016). Spatiotemporal mixed modeling of multi-subject task fmri via method of moments. NeuroImage, 142, 280-292.

Robbins, H. (1980). An empirical bayes estimation problem. Proceedings of the National Academy of Sciences, $77(12), 6988-6989$.

Schwartzman, A., Dougherty, R., Lee, J., Ghahremani, D., \& Taylor, J. E. (2009). Empirical null and false discovery rate analysis in neuroimaging. Neuroimage, 44 (1), 71-82.

Schwartzman, A., \& Telschow, F. (2019). Peak p-values and false discovery rate inference in neuroimaging. NeuroImage, 197, 402-413. 
Smith, S., \& Nichols, T. (2009). Threshold-free cluster enhancement: Addressing problems of smoothing, threshold dependence and localisation in cluster inference. Neuroimage, $44(1), 83-98$.

Smith, S., \& Nichols, T. (2018). Statistical challenges in "big data" human neuroimaging. Neuron, $97(2), 263-268$.

Spisák, T., Spisák, Z., Zunhammer, M., Bingel, U., Smith, S., Nichols, T., \& Kincses, T. (2019). Probabilistic tfce: A generalized combination of cluster size and voxel intensity to increase statistical power. Neuroimage, 185, 12-26.

Waller, L., \& Gotway, C. (2004). Applied spatial statistics for public health data (Vol. 368). John Wiley \& Sons.

Woo, C.-W., Krishnan, A., \& Wager, T. (2014). Cluster-extent based thresholding in fmri analyses: Pitfalls and recommendations. Neuroimage, 91, 412-419.

Yan, C.-G., Wang, X.-D., Zuo, X.-N., \& Zang, Y.-F. (2016). Dpabi: Data processing \& analysis for (resting-state) brain imaging. Neuroinformatics, 14(3), 339-351.

\section{Appendix}

\section{Theoretical properties of eBass primary threshold}

We further provide theoretical results to show the optimality and consistency of the eBass primary threshold estimation.

We first show the eBass solution $\hat{z}_{\theta}$ is optimal with respect to the objective function (1). Specifically, we transform the optimization problem in objective function (1) into a saddle point problem (Eban et al., 2017), and solve it by the cost sensitive methods (Parambath et al., 2014). 
Theorem 1. For $z_{\theta} \in \Omega_{\alpha}$, the eBass estimated primary threshold $\hat{z}_{\theta}$ is optimal with respect to the objective function (1).

Proof. Following the notation in the method section, we define the decision rule $f_{z_{\theta}}: f\left(z_{v}\right) \geq$ $f\left(z_{\theta}\right)$, where $z_{\theta}$ is the primary threshold and $f$ is the mixture density.Let $Y+$ be the set of voxels from the non-null component, and $Y$ - be the voxels for the null component. Then, let $y_{v} \in\{-1,1\}$ be the indicator function where $y_{v}=1$ if $z_{v} \in Y+$ and $y_{v}=-1$ if $z_{v} \in Y-$. We consider the 0-1 loss function of true positive and false positives w.r.t. the empirical Bayes estimated $\hat{f}_{0}, \hat{f}_{1}$. Inherit the notation from Section 2.1, we have

$$
\begin{gathered}
T P\left(f_{z_{\theta}}\right)=\int_{z_{\theta}}^{+\infty} \hat{f}_{1}(t) d t=\sum_{z_{v} \in Y+} \mathbb{1}_{\left[f\left(z_{v}\right) \geq f\left(z_{\theta}\right)\right]}=\sum_{z_{v} \in Y+} 1-l\left(f_{z_{\theta}}, z_{v}, y_{v}\right) \\
F P\left(f_{z_{\theta}}\right)=\int_{z_{\theta}}^{+\infty} \hat{f}_{0}(t) d t=\sum_{z_{v} \in Y_{-}} \mathbb{1}_{\left[f\left(z_{v}\right) \geq f\left(z_{\theta}\right)\right]}=\sum_{z_{v} \in Y_{-}} l\left(f_{z_{\theta}}, z_{v}, y_{v}\right)
\end{gathered}
$$

To bound the above two quantities, replacing the 0-1 loss function by the hinge loss function $l_{h}\left(f_{z_{\theta}}, z_{v}, y_{v}\right):=\max \left(0,1-y_{v}\left(f\left(z_{v}\right)-f\left(z_{\theta}\right)\right)\right.$ leads to the lower bound of true positives $T P_{L}$ and upper bound of false positives $F P_{U}$ at $z_{\theta}$ that satisfies $T P_{L} \leq T P\left(f_{z_{\theta}}\right)$ and $F P_{U} \geq F P\left(f_{z_{\theta}}\right)$ correspondingly.

Once we have the upper bound and lower bound, our objective function 1 can be replaced by a surrogate function $F_{e B}^{*}=\frac{T P_{L}}{|Y+|+T P_{L}+F P_{U}} \leq F_{e B}$. Our goal is to maximize the function $F_{e B}^{*}$ at some given precision. The problem can be written as:

$$
\begin{gathered}
\max \frac{T P_{L}(f)}{|Y+|+T P_{L}(f)+F P_{U}(f)} \\
\text { s.t.TP } P_{L}(f) \geq \alpha\left(T P_{L}(f)+F P_{U}(f)\right)
\end{gathered}
$$


We also replace the notation of the hinge loss function as a shorthand

$$
\ell^{+}(f)=\sum_{z \in Y+} l_{h}\left(f_{z_{\theta}}, z, y\right) ; \ell^{-}(f)=\sum_{z \in Y_{-}} l_{h}\left(f_{z_{\theta}}, z, y\right)
$$

Alternatively, as maximizing $F_{e B}^{*}$ is equivalent to minimizing $\left(F_{e B}^{*}\right)^{-1}$, we rewrite the objective function with $\ell^{+}, \ell^{-}$:

$$
\begin{gathered}
\min \frac{2|Y+|-\ell^{+}(f)+\ell^{-}(f)}{|Y+|-\ell^{+}(f)} \\
\text { s.t. }(1-\alpha)\left(|Y+|-\ell^{+}(f)\right) \geq \alpha \ell^{-}(f),
\end{gathered}
$$

where $\alpha$ is given from the $\widehat{F W E} R_{\text {cluster }}$ constraint.

Straightforwardly, we can write $\phi=|Y+|-\ell^{+}(f)$, and thus the above minimization is equivalent to

$$
\min _{f, \phi} \max _{\lambda} \phi^{-1}\left(1+\ell^{-}(f)\right)-\lambda \phi+\lambda \frac{\alpha}{1-\alpha} \ell^{-}(f),
$$

where $\lambda$ is the Lagrange multiplier. This is similar to optimizing $F_{\beta}$ with weighted loss function established by (Eban et al., 2017) where the Cost-Sensitive Classification algorithm was well explained in (Parambath et al., 2014). Since we restrict the search region to the locally convex neighborhood, the solution of this optimization problem is unique. Therefore, our grid search algorithm guarantees the detection of the unique $z_{\theta}$ and achieves the optimality.

Next, we prove the consistency of the eBass estimated primary threshold $z_{\theta}$. Our proof is mainly built on the fact that the empirical Bayes estimation yields consistent estimators $\hat{f}_{0}, \hat{f}_{1}, \hat{f}$ (Petrone et al., 2014; Robbins, 1980).

Theorem 2. Let $\hat{z}_{\theta}$ be the eBass estimated primary threshold, and we have $\hat{z}_{\theta} \stackrel{p}{\rightarrow} z_{\theta}$.

Proof. We let the test statistics of all voxels follow a marginal distribution $z_{v} \sim f\left(z_{v} \mid \theta_{v}\right)$. 
Let $\boldsymbol{\theta}=\left(\theta_{1}, \theta_{2}, \cdots, \theta_{V}\right)$ be a finite parameter space. Given $\theta_{v} \in \boldsymbol{\theta}$, assume it is independently drawn from a known prior density $\pi(\boldsymbol{\theta})$ corresponds to $z_{v}$.

Since $\boldsymbol{z}=\left(z_{1}, \cdots, z_{V}\right)$ is considered a random sample from $f(\cdot)$, and the marginal distribution of $f(\mathbf{z})=\int_{\boldsymbol{\theta}} \pi(\theta) f(\mathbf{z} \mid \theta) d \theta$ is proportional to the posterior probability, we can calculate the posterior probability $g(\theta \mid \mathbf{z})$.

As the sequence of estimators in $\hat{\boldsymbol{\theta}}$ can be estimated by observed $z_{v}$ with MLE, the empirical Bayes posterior probability is consistent at $\theta_{0}$ in probability, under the common MLE regularity conditions.

By continuous mapping theorem, our $\widehat{T P R}$ and $\widehat{T D R}$ are functions of consistent empirical Bayes estimators. Then we have $\widehat{T P R} \stackrel{p}{\rightarrow} T P R, \widehat{T D R} \stackrel{p}{\rightarrow} T D R$. From Theorem $1, z_{\theta}$ exists and uniquely decided by $T P R$ and $T D R$. Therefore, we have $\hat{z}_{\theta} \stackrel{p}{\rightarrow} z_{\theta}$.

\section{Estimating $\Omega_{\alpha}$ for the step 2 of eBass}

In order to control the cluster level FWER below $\alpha$, we should search $\hat{z}_{\theta}$ on the set $\Omega_{\alpha}$. Here, we describe the procedure to identify the set $\Omega_{\alpha}$ based on the empirical Bayes estimated sensitivity and FDR.

As stated in section 2.2, The cluster level family-wise error rate $\widehat{F W E} R_{c l u s t e r}$ is calculated based on $\int_{\hat{z}_{\theta}}^{+\infty} \hat{f}_{0}(t) d t$. If the total number of false positive voxels $V \int_{\hat{z}_{\theta}}^{+\infty} \hat{f}_{0}(t) d t$ is large at the cut-off of $\hat{z}_{\theta}$, the cluster size of these false positive voxels is likely to be greater than the permutation tests determined cluster-size threshold $K_{\alpha}$. In results, false positive clusters appear in the final results. In order to avoid the false positive cluster, the cut-off $\hat{z}_{\theta}$ is required to prohibit forming large clusters.

Specifically, we denote the estimated number of false positive voxels using a cut-off $\hat{z}_{\theta}$ by $m_{f p}\left(\hat{z}_{\theta}\right)=V \hat{\pi}_{0} \int_{\hat{z}_{\theta}}^{+\infty} \hat{f}_{0}(t) d t$. We next compute the upper bound of the cluster size based on the combinatorial probability that these $m_{f p}\left(\hat{z}_{\theta}\right)$ false positive supra-threshold voxels can form a contiguous non-trivial cluster in the three-dimensional brain space. We define the 
search domain for $\hat{z}_{\theta}$ by $\Omega_{\alpha}=\left\{z_{\theta}: \operatorname{Sup}\left\{\mu\left(m_{f p}\left(z_{\theta}\right)\right)\right\}<K_{\alpha}\right\}$, where $\mu$ is the cardinality measure of any set of contiguous voxels formed by $m_{f p}\left(z_{\theta}\right)$ in the brain space. In practice, the direct calculation of $\operatorname{Sup}\left\{\mu\left(m_{f p}\left(z_{\theta}\right)\right)\right\}$ is intractable. We resort to permutation based techniques to approximate $\operatorname{Sup}\left\{\mu\left(m_{f p}\left(z_{\theta}\right)\right)\right\}$.

In each permutation, the random shuffling of subject labels hypothetically produces a $\hat{f}_{0}^{*}$ distribution and an $\alpha_{p}$ level is chosen to control the permutation test FWER. Since we are able to theoretically calculate the $m_{f p}\left(\hat{z}_{\theta}\right)$ based on empirical Bayes estimated $\hat{f}_{0}, \hat{f}_{1}$, we consider 1) an $\alpha_{1}$ for permutation bound that controls the FWER among all supra-threshold voxels, 2) an $\alpha_{2}$ for false positive cluster bound controls the FWER for estiamted false positive voxels. Commonly, the widely used $5 \% \alpha_{p}$ level is unadjusted and that $\alpha_{p}=\alpha_{1}$. The adjustment according based on the definition of $\alpha_{1}$ and $\alpha_{2}$ is $\left(1-\alpha_{1}\right)\left(1-\alpha_{2}\right)=1-\alpha_{p}$. For $\alpha_{2}$, it is calculated by the top $m_{f p}\left(\hat{z}_{\theta}\right)$ voxels on both tail-end of $\hat{f}_{0}^{*}$ and randomly choose $m_{f p}\left(\hat{z}_{\theta}\right)$ from those extreme observations to form false discovery clusters. The adjustment indicates that when $\alpha_{2} \rightarrow 0,1-\alpha_{1} \approx 1-\alpha_{p}$. In other words, we need to take the cluster size corresponds to $\max \left\{\alpha_{2}\right\}$ (or other levels based on the adjustment) to estimate $C_{\alpha, z_{\theta}^{*}}$, and then we can estimate the support $\Omega_{\alpha}$.

We summarize the procedure as follows:

Step 1: For a cut-off $z_{\theta}^{*}$, we calculate $m_{f p}\left(z_{\theta}^{*}\right)$, the number of false discoveries based on the empirical Bayes estimated parameters.

Step 2: Compute $K_{\alpha}$ by shuffling subject labels at the cut-off $z_{\theta}^{*}$.

Step 3: Shuffle the subject labels for $J$ times. At each iteration $j$ we select $m_{f p}\left(z_{\theta}^{*}\right)$ voxels with the largest test statistics and a similar dependence structure.

Step 4: Let $C_{\alpha, z_{\theta}^{*}}$ denote the maximum (or other adjusted $\alpha$ level) cluster size across the $J$ permutations in step 3 . 
Step 5: $z_{\theta}^{*} \in \Omega_{\alpha}$ if $C_{\alpha, z_{\theta}^{*}}<K_{\alpha}$.

In practice, we find both $C_{\alpha, z_{\theta}^{*}}, K_{\alpha}$ are monotonously decreasing with $z_{\theta}^{*}$ while the decreasing speed of $C_{\alpha, z_{\theta}^{*}}$ is faster. Therefore, $\Omega_{\alpha}$ is often a continuous domain.

\section{Comparison between Cluster-extent Methods and Voxel-wise In- ference}

Although we focus on the two-step cluster-wise inference in the simulation study, we further compare the cluster-extent methods with TFCE - one of the most popular voxel-extent inference. The TFCE generates voxel-level corrected $p$ values instead of considering clusters as a whole, thus the cluster-wise FWER is not applicable to this method. Similarly, as in cluster-wise inference we assume all supra-threshold voxel-formed clusters are significant, we compare the voxel-wise sensitivity and FDR of primary thresholding step specifically with TFCE.

The $2 \mathrm{D}$ image has the same dimension with $V=100 \times 100=10,000$ voxels. The truth consists of four identical squared areas that are placed in the center of the image with equal distance (roughly same as the side of the square). Different from the images in the simulation section, we apply the smoothing step after adding the true signals to the original image. In this way, the underlying truth have an irregular shape on the margin, and the strength of signal decreases steady from center to margin. The total number of true significant voxels is 900 . We perform the simulation study on the set of images have $\mathrm{ES}=0.6$, smoothed with FWHM=8mm, 30 subjects per group.

We perform the two-step cluster-wise inference with eBass, BH-FDR correction, $p<$ 0.001, and $p<0.01$. We also apply TFCE voxel-wise inference on the images under this setting. Similarly, we evaluate the performance of methods by their voxel-wise sensitivity, FDR, and cluster-wise FWER. We exhibit the simulation results in Table 2. 
From the example above, we find out that when the significant clusters have blurred edges and close to each other, the voxel-extent methods would have a significant increase (up to $50 \%$ ) in vFDR comparing to cluster-extent methods. When the ES and sample size are low to medium, the voxel-extent method (e.g., TFCE) tends to outperform cluster-extent methods on sensitivity by $5-30 \%$ in this type of images who have clear edges, while the vFDR remains about the same.

\begin{tabular}{cccccc}
\hline & eBass & BH-FDR & $<0.001$ & $<0.01$ & TFCE \\
\hline Primary Threshold & $0.003 \pm 0.0029$ & $0.0063 \pm 0.0001$ & 0.001 & 0.01 & NA \\
Sensitivity & $0.9996 \pm 0.0007$ & $0.9998 \pm 0.0004$ & $0.9991 \pm 0.001$ & $0.9998 \pm 0.0004$ & 1 \\
vFDR & $0.2469 \pm 0.025$ & $0.2644 \pm 0.0129$ & $0.2200 \pm 0.0096$ & $0.2767 \pm 0.0116$ & $0.3602 \pm 0.0142$ \\
cFWER & 0 & 0 & 0 & 0 & NA \\
\hline
\end{tabular}

Table 2. Performance comparison between cluster-extent methods and voxel-wise thresholding method. 30 subjects per arm with $\mathrm{ES}=0.6$, smooothed with Gaussian kernel $\mathrm{FWHM}=8 \mathrm{~mm}$.

\section{Primary Thresholds for Real Data}

\begin{tabular}{ccc}
\hline eBass & Hard Threshold $\mathrm{p}<0.001$ & Hard Threshold $\mathrm{p}<0.01$ \\
\hline $0.0018(\approx 154)$ & $0.001(105)$ & $0.01(853)$ \\
\hline
\end{tabular}

Table 3. Primary thresholds and corresponding cluster-extent thresholds 